\title{
THE ROLE OF BINARIES IN CLUSTER DYNAMICS
}

\author{
D. C. HEGGIE \\ Trinity College, Cambridge and Institute of Astronomy, Cambridge, U.K.
}

\begin{abstract}
There is considerable empirical evidence on the behaviour of binary stars in computer simulations of isolated star clusters. Even when there are no binaries initially they form quickly if the mass spectrum of the stars is reasonably realistic. These phenomena are discussed by a combination of analytical and numerical techniques.
\end{abstract}

\section{Introduction}

There is now considerable empirical evidence on the behaviour of binary stars in computer simulations of isolated star clusters. In particular, Aarseth (1972) showed that, even when there are no binaries initially, they form quite quickly if the mass spectrum of the stars is reasonably realistic. Even for large clusters with up to 500 members, it is found that, within a few mean crossing times, one or two binaries have taken possession of most of the binding energy of the cluster. It takes longer if all masses are equal. Also, the evolution of these energetic binaries is accompanied by energetic escape of stars from the cluster.

In a thesis (Heggie, 1972) and also in a paper now under preparation by the author, these and other phenomena are discussed in detail by a combination of analytic and numerical techniques. Here some of the results will be summarized without detailed derivation, in order to emphasize qualitatively the implications of this work for our understanding of the dynamics of star clusters.

One question of importance concerns the relation between the evolution of the binaries and that of the cluster. Does the observed development of energetic binaries merely follow in response to the evolution of the cluster by other processes such as collisional relaxation, as held by Hénon (1972), or does the energetic behaviour of the binaries itself exert a substantial influence on changes in the structure of the cluster?

\section{Binaries in Equilibrium}

Arguing by analogy with the theory of chemical equilibrium, several authors (e.g. Jeans, 1929) have suggested that the distribution of binaries should relax to the Boltzmann form. The resulting distributions of $\varepsilon$, the pair binding energy, and of the eccentricity, $e$, take the forms

$$
\begin{aligned}
& f(\varepsilon) \propto \varepsilon^{-5 / 2} \exp (\varepsilon / k T), \\
& f(e)=2 e,
\end{aligned}
$$

respectively, where $\frac{3}{2} k T$ is the mean random kinetic energy of the stars in the cluster. Equation (2) fits experimental data very well, one example being the result of Aarseth and Hills (1972), but Ambartsumian (1937) pointed out that it follows from many 
distributions besides the Boltzmann. The energy distribution (1) is in conflict with experiment, and the singularity for $\varepsilon \uparrow \infty$ is physically implausible.

Certainly the Boltzmann distribution is incorrect, but in addition the testimony of computer experiments is that, for energetic binaries at least, the phenomenon is not one of equilibrium. Interactions with other stars are crucial, and they lead to the evolution and, sometimes, the disruption of binaries. The analogy with atomic or nuclear collision processes is so close that the language of 'cross-sections', 'resonances', and so on, is appropriate.

\section{Soft Binaries}

The first case to be considered occurs when $\varepsilon \ll k T$. The internal energy of the binary is much less than the energy of the relative motion of the third body before collision, and such a binary is henceforth termed 'soft'. An equipartition argument suggests that, on average, kinetic energy is transferred to the internal energy of the binary, and so such binaries tend to disrupt (Gurevich and Levin, 1950).

For theoretical calculations the encounter may be treated as impulsive. Then the cumulative cross-section for energy changes greater than a certain amount may be computed for comparison with the results of specially-designed numerical experiments. The agreement is satisfactory, and also the theory confirms the conclusion of the above physical argument that soft binaries exhibit a tendency to disruption.

Soft pairs also tend to an approximate equilibrium distribution, the time scale for its attainment being about $0.06 N(\varepsilon / k T) t_{\mathrm{cr}}$, where $N$ is the number of particles in the system, and $t_{\mathrm{cr}}$ is the mean crossing time. Since energy changes of order only $\varepsilon$ are required for disruption of a soft pair with energy $\varepsilon$, this time is generally much less than a mean Chandrasekhar relaxation time, which is the time scale for changes of order $k T$.

\section{Hard Binaries}

The case when $\varepsilon \gg k T$, that of 'hard' binaries, is the other case for consideration. If the change in binding energy of the binary during the encounter is large and positive, the third body receeds with great energy afterwards. This is one source of the energetic escapers noted by Aarseth (1972) and others (e.g. Hayli, 1972). If the change in energy of the binary is negative and large enough, the third body will become bound to the binary. One component of the original binary may be ejected immediately in an 'exchange' event. Alternatively, from the work of Agekyan and Anosova $(1967,1968)$ it is known that most bound triple systems end with the release of an energetic escaper. The net effect of all these processes is that hard binaries tend to become harder.

Theory is more difficult than for soft pairs, but it can be developed roughly for ordinary close encounters and exchange events, and perturbation techniques are applicable for distant encounters. For example, it may be shown that binaries tend to form from the most massive stars.

The most difficult theory is that of bound triple systems. The rate at which they form can be calculated roughly, and then a detailed balance argument may be applied to 
find how fast these triple systems disrupt, if it is assumed that a bound triple system eventually 'forgets' most of its initial conditions except for the values of the classical integrals. This may be likened to the 'Bohr assumption' of nuclear physics (e.g. Blatt and Weisskopf, 1952). Here it is very successful in predicting the distribution of the energy of the escaping particle, as comparison with the results of Szebehely (1972) shows.

Wide encounters with a hard binary have one interesting property. Changes in the eccentricity are generally much bigger than changes in energy, and they are systematic if the third body is loosely bound to the binary. The latter phenomenon was noted in $N$-body experiments by Aarseth (1972).

It turns out that the average rate at which a hard binary gains energy is approximately independent of the binding energy of the binary. This result follows from two facts. First, the energy changes are typically of order $\varepsilon$ itself, as a result of close encounters. Second, the total cross-section for close encounters is proportional to $a$, where $a$ is the semimajor axis. It is not proportional to $a^{2}$, the area of the binary, because approaching particles converge towards a hard binary. Hence the rate of change of binding energy is proportional to $a \varepsilon$, which is independent of the energy. Note that this result applies to the average rate of change of energy; if a binary is too hard, a close encounter is so rare that none is likely to occur during the whole lifetime of the cluster.

\section{Applications}

It can be shown that the total time taken for the hard binaries to form and then absorb an amount of energy equal to the total energy of the cluster is about $\left(N^{2} / B\right) t_{\mathrm{cr}}$, where $B$ depends very sensitively on the structure of the cluster, but a value of about 100 is not untypical. Now the time taken for collisional relaxation to exchange the same amount of energy is about $(N / 50 \log N) t_{\mathrm{cr}}$ (Chandrasekhar, 1942). This is generally much less, and so we conclude that the evolution of binaries does not have a significant energetic effect on the overall evolution of the cluster provided that $N$ is not very small. For example, during the final evolution of the core, which may be treated roughly as a small $\mathrm{N}$-body system within the cluster, binary evolution is very important. Experimental results discussed by Hénon (1972) already pointed to the fact that binary evolution has negligible effect on the evolution of the cluster in its early stages, before core formation.

In each star cluster only one or two very hard binaries are produced. Yet, the proportion of visual binaries in the neighbourhood of the Sun is very high (van de Kamp, 1971). We conclude that only a few of these can have been formed by dynamical processes in star clusters, although such an explanation has been proposed (Gurevich and Levin, 1950; Kumar, 1972). The rest must have formed through processes occurring early in the lifetime of the stars, possibly at birth as discussed by Larson (1972) and others. Hence it may be expected that star clusters initially should contain large numbers of binaries.

If this is so, the previous estimate of the time taken for hard binaries to absorb the 
energy of the cluster is in need of revision. It becomes about $\left(N^{2} / 70 N_{b}(0)\right) t_{\mathrm{cr}}$, and if $N_{b}(0)$, the initial number of hard binaries, is comparable with $N$, then this time is of the same order as the relaxation time. It may be expected then that the evolution of hard binaries must play as important a part in the evolution of the cluster as collisional relaxation. The effects will be qualitatively different, since two-body encounters are elastic, while those involving binaries are typically superelastic.

It is urged that attention be paid in future to the dynamics of star clusters initially containing many binaries. They may be astrophysically more relevant than clusters without binaries, and their dynamics may be different.

\section{Acknowledgements}

I am grateful to several people, but especially to Dr S. J. Aarseth, for innumerable discussions on binaries, to Trinity College, Cambridge, for a Research Fellowship, and to the Institute of Astronomy, Cambridge, for its hospitality.

\section{References}

Aarseth, S. J.: 1972, in M. Lecar (ed.), Gravitational N-Body Problem, D. Reidel, Dordrecht, The Netherlands, p. 88.

Aarseth, S. J. and Hills, J. G.: 1972, Astron. Astrophys. 21, 255-63.

Agekyan, T. A. and Anosova, Zh. P.: 1967, Astron. Zh. 44, 1261-73.

Agekyan, T. A. and Anosova, Zh. P.: 1968, Astrofiz. 4, 31-40.

Ambartsumian, V. A.: 1937, Astron. Zh. 14, 207-19.

Blatt, J. M. and Weisskopf, V. F.: 1952, Theoretical Nuclear Physics, Wiley, London, p. 340.

Chandrasekhar, S. : 1942, Principles of Stellar Dynamics, University of Chicago Press, Chicago, p. 201.

Gurevich, L. E. and Levin, B. Yu.: 1950, Astron. Zh. 27, 273-84.

Hayli, A.: 1972, in M. Lecar (ed.), Gravitational N-Body Problem, D. Reidel, Dordrecht, The Netherlands, p. 73.

Heggie, D. C.: 1972, Ph.D. Thesis, Cambridge University (unpublished).

Hénon, M.: 1972, in M. Lecar (ed.), Gravitational N-Body Problem, D. Reidel, Dordrecht, The Netherlands, p. 44.

Jeans, J. H.: 1929, Astronomy and Cosmogony, 2nd ed., Cambridge University Press, p. 302.

Kumar, S. S.: 1972, Astrophys. Space Sci. 17, 453-58.

Larson, R. B.: 1972, Monthly Notices Roy. Astron. Soc. 156, 437-58.

Szebehely, V.: 1972, Celes. Mech. 6, 84-107.

van de Kamp, P.: 1971, Ann. Rev. Astron. Astrophys. 9,

\section{DISCUSSION}

M. Lecar: Do you think that in actual globular clusters, initial binaries (i.e. binaries present at birth) significantly affect the time scale of the dynamical evolution of the cluster?

D. C. Heggie: Provided that the initial number of binaries that are hard (but not too hard) is significant in comparison with the number of stars, then their effect also will be significant. The initial number depends on details of the process by which binaries form, but the evidence suggests that the total number of all hard binaries will be large.

M. Hénon: You said that the time needed by a hard binary to swallow the whole energy of the system is of order $N^{2} t_{\mathrm{cr}}$, therefore, much larger than the relaxation time. But is not it true that in numerical 
experiments like those of Dr Aarseth a central hard binary usually absorbs a large fraction of the total energy?

D. C. Heggie: The statement on the time scale is true provided that the structure of the cluster is not too 'extreme'. For example, it is spatially extremely inhomogeneous in the late stages of core evolution when these hard binaries are observed to form. Alternatively, you can regard the core as a small $n$-body system within the cluster, and then the formula shows that binary evolution is quite rapid. 